\title{
Temporal reasoning about composite and/or periodic events
}

\author{
PAOLO TERENZIANI*†, LUCA ANSELMA
}

† Dipartimento di Informatica, Università del Piemonte Orientale ‘Amedeo Avogadro’, via
Bellini 25/g, Alessandria, Italy. Phone: +39 0131360174 Fax: +39 0131360198 - Email:
terenz@mfn.unipmn.it

†Dipartimento di Informatica, Università di Torino, Corso Svizzera 185, 10149 Torino, Italy. Phone: +390116706821 Fax:+39011751603 - Email: anselma@di.unito.it

In many application areas, including planning, workflow, guideline and protocol management, the description of the domain involves composite and/or periodic events, mutually related by temporal constraints on the execution order. Such events represent 'classes', since they can be instantiated to specific executions of the plan, guideline etc., and each execution must 'respect' the temporal constraints imposed on the corresponding classes. The main objective of our work is that of proposing an approach dealing with the above-mentioned temporal phenomena. To achieve such an objective, we propose a tractable domain-independent temporal reasoner. This enhances the generality of our approach, which provides a domain-independent module which can be integrated with other software tools to solve temporal problems in specific domains.

From the methodological point of view, we first devise a representation formalism coping with the aforesaid phenomena, and then we describe temporal constraint propagation algorithms to deal with constraint inheritance and to perform temporal consistency checking. The representation formalism has been designed carefully, to obtain algorithms that are both complete and tractable. Finally, the paper also shows experimental results, including an application of our approach to clinical guidelines, evaluating their impact on future applications and research activities.

\section{Introduction}

Time surely plays a fundamental role when modelling reality, and reasoning about time is essential in most intelligent activities. Thus, in the field of Artificial Intelligence (henceforth, AI) many formalisms and reasoning techniques have been defined to deal with time-related phenomena. Roughly speaking, one could identify two different mainstreams (see Terenziani (2003b)).

The first mainstream is mainly devoted to the definition of formalisms general enough to represent a wide range of temporal phenomena. It aims at dealing with the dynamic aspects of the world, at describing the internal structure of actions and events occurring over the world, and at modelling how the world changes in response to such actions. Although very different formalisms have been devised to such a purpose, most of these approaches proposed first order, modal, or nonmonotonic logical formalisms.

The second mainstream consists of approaches which we will call 'constraint-based' approaches. It is mainly focused on the definition of representation formalisms and of reasoning techniques to deal specifically with temporal constraints between temporal entities per se, without regard to the events and states which take place over such entities. By focusing on a more restricted problem, one could obtain higher efficiency than general-purpose approaches. In fact, it could be possible to 
define specialised reasoning techniques that make inferences in a more efficient way than, e.g., a standard theorem prover for the first-order logic. The approach we are going to describe in this paper belongs to the mainstream of constraint-based approaches.

Since the 1980s, many AI researchers have recognized the importance of designing and implementing specialized approaches in order to perform temporal reasoning on different types of temporal constraints (Vilain et al. 1990, Allen 1991, Vila 1994). Such approaches are mostly conceived to define domain-independent knowledge servers which temporal constraint propagation problems can be delegated to, and which possibly have to be coupled with other modules (e.g., a planner) to solve complex problems.

The aim towards specialization led these approaches to focus on specific classes of constraints (e.g., qualitative constraints (Allen 1983), quantitative constraints (Dean and McDermott 1987)) between time points or intervals, and to devote great attention to the trade-off between the expressiveness of the formalisms and the computational complexity of (possibly complete) temporal reasoning algorithms operating on them. More recently, the focus has also been extended to the treatment of user-defined periodic/repeated events and/or granularities (e.g., (Ladkin 1986, Ligozat 1991, Morris et al. 1993, Loganantharaj and Gimbrone 1995, Cukierman and Delgrande 1998, Bettini and DeSibi 1999, Bettini et al. 2002, Combi et al. 2004)) and the integration of different kinds of constraints (e.g., qualitative and quantitative constraints (Meiri 1991), pointduration constraints (Navarrete et al. 2003)).

However, despite the large amount of valuable works in the area, there still seems to be a gap between the range of phenomena covered by current constraint-based temporal reasoning approaches and the needs arising from many real-world application areas, including planning and management of protocols and guidelines.

In particular, in such domains:

(1) qualitative and quantitative constraints, as well as repeated/periodic events need to be considered at the same time;

(2) a structured representation of complex events (in terms of part-of relations) is always employed, to support top-down refinement and a structured description of the domain knowledge;

(3) the distinction between classes of actions (e.g. an action in a general plan) and instances of such actions (e.g., the specific execution of an action in a plan) has to be supported.

While issue (1) explicitly mentions temporal constraints, it is important to note that also issues (2) and (3) have temporal side-effects which a temporal reasoner has to cope with. For instance, partof relations involve temporal containment between the composite action and its components, and instance-of relations between actions involve the fact that the constraints between classes must be 'respected' by the corresponding instances.

Thus, when working in complex domains such as planning and protocol/guideline management, if one wishes to provide a knowledge server which reasoning about temporal constraints can be delegated to, she needs to devise a homogeneous approach able to cover all the above issues. Unfortunately, the fact that data stored in a (temporal constraint) knowledge base are often associated with underlying semantic assumptions, and that there are subtle interplays between the issues (1)-(3) above makes this task quite difficult, and no approach in the literature covers all of them simultaneously.

In the rest of this paper, we propose an initial step towards such a task.

In section 2, we introduce the phenomena to be addressed by our constraint-based temporal manager, sketching the problems to be faced and the strategy we used to devise our approach. In section 3, we describe the high-level language we introduce in order to represent the above phenomena, and the low-level language we use to map the high-level description in an internal form, on which temporal reasoning (via constraint propagation) can be performed. In section 4 we deal with constraint propagation, considering constraints between classes only. In section 5, we extend our temporal reasoning approach to consider also inheritance and classes + instances 
temporal reasoning. Two different algorithms are introduced, depending on the underlying semantic assumptions.

Despite the wide range of phenomena we investigated, we aimed at retaining tractability and completeness of the reasoning process, and this fact led us to introduce some restrictions on the expressiveness of the temporal language and some simplifying assumptions. In section 6 , besides discussing applications and future and related works, we sketch some extensions to our current approach in order to overcome some of its limitations.

\section{An introduction to the problem}

In order to introduce the range of temporal phenomena we aim to deal with, let us start from a practical example, concerning a clinical guideline about the treatment of multiple mieloma:

(Ex. 1) The therapy for multiple mieloma is made by six cycles of five days treatment, each one followed by a delay of 23 days (for a total time of 24 weeks). Within each cycle of five days, two inner cycles can be distinguished: the melphalan treatment, to be provided twice a day, for each of the five days, and the prednisone treatment, to be provided once a day, for each of the five days. These two treatments must be performed in parallel.

Of course, such a therapy might have been executed (or being in execution) on different patients at different times, such as in Ex. 1':

(Ex. 1') Melphalan was given to John on Jan 13, 2004, between 12:00 and 12:15 and then from 18:00-18:10 to 18:20. Prednisone was given to John on Jan 13, 2004, between the two administrations of melphalan...

In order to deal with the temporal constraints in Ex.1 and Ex.1', one has also to consider, besides qualitative and quantitative temporal constraints between actions:

(i) periodic actions (i.e., actions to be repeated at periodic times; e.g., administering melphalan) and temporal constraints between them;

(ii) composite actions (e.g., the treatment of multiple mieloma);

(iii) classes of actions (e.g., actions in Ex.1) vs instances of actions (e.g., actions in Ex.1').

The development of a temporal reasoning approach dealing in an integrated way with all such issues requires the analysis of different problems. We sketch such problems in an incremental way: we start from the treatment of constraint inheritance in the basic case, and then we describe the additional issues to be faced when dealing with periodic/repeated actions and with composite actions.

\subsection{Temporal Constraints Inheritance}

Usually, general plans (guidelines, protocols, workflows) contain temporal constraints between (classes of) events. Constraints about durations, delays and precedences are typical examples. Consider also, e.g., Ex.2:

Ex.2. Test reservation must be done between one and seven days before the laboratory test.

Of course, these are temporal constraints between classes of events, which might be instantiated many times, for different instantiations of the classes of events. In such a context, checking whether each instantiation 'respects' the temporal constraints described in the plan (guideline, protocol, workflow) constitutes a fundamental task. The basic problem is quite general. At a given point in time (say Reference Time, henceforth $\underline{\mathrm{RT}}$ - in the rest of the paper, as in many approaches, we assume that RT is equal to 'now') the knowledge base of the system contains a set of constraints between classes of events, as well as a set of instances which might be imprecisely located in time (via a set of temporal constraints). Depending on the domain, one can assume full observability (i.e., all the instances of events that occur are observed and are inserted into the 
knowledge base) or not, and, in certain cases, even considering instances located after RT (i.e., future events) makes sense. The problem is to check whether the instances are located in time in such a way that they respect the temporal constraints imposed by their classes. In other words, the temporal constraints between classes must be 'inherited' by instances, and the union of the given constraints between instances (consider, e.g., Ex.1') and the inherited constraints must be consistent.

The first step in the definition of a temporal reasoner coping with such an issue involves the recognition of the 'relational' character of binary temporal constraints between classes. In fact, such constraints have to be inherited only by 'corresponding' pairs of instances of the related classes. For instance, Ex.2 does not mean that all the reservations must be done before all laboratory tests, but that each reservation must be one to seven days before the correlated (Morris et al. 1993, Terenziani 1997) instance of laboratory test. For instance, in figure 1, we show a consistent scenario for the constraints in Ex.2, in which $R 1$ is correlated to $T 1$ and $R 2$ to $T 2$ (notice that the scenario is consistent even if there is a test -namely $T 1$ - that precedes a reservation $-R 2$ ).

\section{insert figure 1 about here.}

In general, different rules could be devised to infer whether two instances of events are correlated or not, depending on the specific context and domain. Modelling correlation is outside the goals of this paper (further discussions on correlation are in (Morris et al. 1993, Terenziani 1997)).

Moreover, one should also take into account the fact that plans (or protocols, or guidelines) have a 'predictive' role. For instance, given Ex.2, if one has observed a given instance $R 1$ of the (class of) event Reservation, one expects to observe an instance of $T 1$ of the correlated Laboratory Test within one and seven days. The impact of such a predictive character on the temporal reasoning algorithm varies depending on the semantic assumptions concerning observability and the treatment of future events. In domains where one is certain to have a full and complete observability of events, the consistency check of the temporal constraints must take into account 'prediction', since not having observed a given instance of event in a given time lapse may indicate an inconsistency. The problem is more complex in the (more common) case where full observability only concerns times before RT (e.g., if proactive updates are not allowed, so that no event in the future can be instantiated). In such a case, the knowledge base only contains all and only the instances that occurred at a time which is before or equal to the time when the temporal reasoner is activated (i.e., RT). Thus, not having observed a predicted instance does not raise an inconsistency (since such an instance could be observed in the future), unless the temporal constraints impose that it should have started before RT.

\subsection{Periodic events}

Repeated events are widespread in many application domains. In many cases (consider, e.g., clinical therapies), repeated events are periodic, since they must be executed at regular (i.e., periodic) times. In a broad sense, thus, periodic events are special kinds of classes of events, i.e., classes whose instances must respect a given periodic temporal pattern. In such a sense, constraints on the periodicity of repetitions are constraints that must be 'inherited' by instances. However, it is a 'non-classical' form of inheritance. In fact, while constraints about durations, delays and precedences regard single instances (durations) or pairs of instances (delays, precedences), periodicity constraints concern sets of instances, imposing constraints on their cardinalities and on the temporal patterns they have to respect.

In such a context, determining the correct association (Morris et al. 1993) between (instances of) events and periodicities is a critical issue. Consider, for instance, the constraint (class level) in Ex. 
3 , and the situation shown in figure 2 , where dotted intervals represent uncertainty about the temporal location of the instances of the event $A$ (melphalan treatment).

Ex.3 Melphalan treatment must be done twice each day before prednisone.

insert figure 2 about here.

In such a situation, the problem is that of determining an association between melphalan treatment's instances and days, so that the pattern in Ex.3 (i.e., two events each day) is respected (e.g., $M 1$ and $M 2$ in D1, M3 and $M 4$ in D2, and M5 and M6 in D3 is a possible solution). Only after a correct association is found, the precedence constraints between melphalan and prednisone can be inherited.

\subsection{Composite events}

In general, plan/guideline/protocol formalisms consider the notion of composite events, to support top-down refinement in the description of the domain. This is a critical feature in most real-world applications, where the domain description consists of hundreds of events. However, the treatment of composite events has several temporal implications.

The first, obvious, one is the temporal constraint that the temporal extent of a composite action is the minimal convex time interval covering the temporal extent of its components (we do not deal with non-convex intervals in this paper).

A less obvious side-effect arises in combination with the treatment of classes and instances of composite actions. In fact, composite actions are 'abstractions' which are used in the description of the domain (e.g., of a plan, or of a guideline). On the other hand, in most cases, no instances of composite actions are observed (i.e., inserted in the knowledge base). For instance, given the (part of) guideline in Ex.1 and its execution on John (Ex.1'), we will not find any instance of 'mieloma treatment' in the clinical record of John, but just instances of the atomic actions 'administering melphalan' and 'administering prednisone'. Nevertheless, temporal constraints between composite actions must be taken into account by the temporal reasoner (consider, e.g., Ex.4).

Ex.4 A follow-up therapy must be done after the mieloma treatment.

This means that the temporal reasoner must be able to properly map the temporal constraints about composite events onto their composing atomic events.

Finally, notice that the interplay between composite and periodic events might be complex to represent and manage. In fact, in the case of a composite periodic event, the temporal pattern regards the components, which may, recursively, be composite and/or periodic events. For instance, consider again Ex.1. In Ex. 1, the instances of the melphalan treatment must respect the temporal pattern 'twice a day, for five days', but such a pattern must be repeated for six cycles, each one followed by a delay of 23 days, since the melphalan treatment is part of the general therapy for multiple mieloma.

\subsection{A two-layer temporal manager}

The final goal of our approach is that of devising a tractable knowledge server (constraintpropagation-based temporal reasoner) which deals with all the above-mentioned phenomena, thus offering a support for:

(i) explicitly representing a description of the domain in terms of part-of and instance-of relations;

(ii) explicitly representing the temporal constraints between classes of events, including periodic repetitions, and the temporal constraints between instances of events, including correlation relations; 
(iii) reasoning about inheritance of temporal constraints and performing consistency checking.

Some of the above issues have been treated in an ad-hoc way in the planning/guideline context, embodying their treatment in the code for planning/guideline management. On the other hand, we think that a general domain-independent module coping in an integrated way with all of them constitutes a relevant advance, providing a temporal knowledge server which acts as an independent module which temporal problems in different domains may be delegated to (so that, e.g., one can focus only on the problems concerning specific guidelines, without taking care of the treatment of temporal issues).

The strategy we chose to adopt in order to achieve our goal is that of devising a two-layer approach:

(1) the high-level layer provides a high-level language to represent the above-mentioned temporal phenomena and a temporal consistency checking facility, providing a highlevel easy-to-use interface to users and applications, so that most of the complexity of the approach can be hidden to the user;

(2) the low-level layer consists of an internal representation of the temporal constraints, on which temporal constraint propagation algorithms operate.

At the high-level layer, we designed our language in such a way that tractable temporal reasoning can be supported. At the low-level layer, we chose to exploit as much as possible STP (Simple Temporal Problem (Dechter et al. 1991)), a standard temporal reasoning framework which has very interesting computational properties (see section 3.1). Obviously, the gap between the highlevel layer language on one side and STP on the other is very large. Filling such a gap involves the design of suitable temporal reasoning algorithms to cope with the problems discussed in sections 2.1, 2.2 and 2.3 above, as well as an extension of the STP framework itself (to consider labelled trees of STPs -see section 3.4). Filling such a gap is the main contribution of our approach, which uses STP as an 'assembly language' and builds an expressive 'high-level temporal reasoning framework' on top of it. In some sense, we thus think that our contribution is analogous, in the temporal reasoning area, to the design of a new language in the programming area. New languages are finally compiled/interpreted into some 'standard' assembly language, but they are nevertheless introduced in order to provide high-level tools to make easier the work of programmers.

\section{An integrated approach to temporal reasoning about classes and instances of events}

In section 3.1, we briefly discuss STP. Then, we describe the languages we propose in order to represent temporal constraints between instances (section 3.2) and classes (section 3.3). In section 3.4, we introduce STP-trees, an extension to the STP framework to deal also with repeated/periodic actions.

\subsection{STP}

The STP framework is based on the notion of bound-on-difference constraints. A bound on difference is a linear inequality of the form $d_{1} \leq X-Y \leq d_{2}$ (also strict inequalities are allowed). The variables in a bound on difference may correspond to time points (and extremes of time intervals). Thus, a bound on difference $d_{1} \leq X-Y \leq d_{2}$ may have the following temporal interpretation: the temporal distance between the time points (endpoints of time intervals in our case) $X$ and $Y$ is between $d_{1}$ and $d_{2}\left(d_{1}, d_{2} \in \mathfrak{R}\right)$. A temporal knowledge base is a conjunction of bound-ondifference constraints between variables representing the starting/ending points of events (called

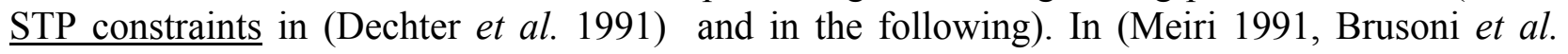
1997) it is shown how different types of temporal constraints can be easily mapped to STP constraints (e.g., this is the case for all the temporal constraints in sections 3.2 and 3.3 below). In 
particular, all the qualitative constraints of the Continuous Interval Algebra (i.e., the subset of relations of Allen's Interval Algebra which can be mapped onto conjunctions of constraints between points, excluding inequality (Vila 1994)) can be mapped onto STP constraints, as well as precise and imprecise dates, durations and delays*. A set of STP constraints on a set K of variables can be represented in terms of a graph called temporal constraint network. The strictest constraints between each pair of variables (nodes) in K (i.e., the minimal network) can be obtained using a standard all-to-all shortest path algorithm such as, e.g., Floyd-Warshall's one, which operates in a time cubic in the number of variables (nodes in the graph) and is correct and complete for STP constraints (Dechter et al. 1991). Moreover, it can also be adapted to check consistency (the temporal constraint network is consistent iff its minimal network does not contain negative cycles (Dechter et al. 1991)). Finally, conjunctions of STP constraints support efficient query answering (Brusoni et al. 1995) and incremental insertion of new constraints (Console and Terenziani 1999).

\subsection{Language for temporal constraints between instances of events (ITL)}

Time points are the basic notion in our approach. A time interval I is a convex set of points between a starting $(\operatorname{Start}(I))$ and an ending $(\operatorname{End}(I))$ point. Different types of temporal constraints can involve instances of events.

\subsubsection{Temporal constraint language for instances}

Our high-level constraint language over instances (called ITL) has been designed in such a way that all high-level constructs map onto conjunctions of STP constraints.

In ITL dates can be expressed using the high-level predicate date(E,L1,U1,L2,U2), stating that the starting point of $E$ is a time point temporally located between $L 1$ and $U 1(L 1 \leq U 1)$ and its ending point is a time point between $L 2$ and $U 2(L 2 \leq U 2)$. Exact dates can be simply modelled using $L 1=U 1$ and $L 2=U 2$; unknown extremes can be represented as $-\infty$ and $+\infty$.

Durations are represented in ITL by the predicate duration(E,L1,U1), stating that $L 1$ and $U 1$ are the minimum and maximum durations of $E$ respectively $(L 1 \leq U 1)$. Delays represent the temporal distances between pairs of instances (e.g., $T 1$ started one to two days after the end of $R l$ ) and are represented in ITL by the predicate $\operatorname{delay}(\mathbf{P 1}, \mathbf{P 2}, \mathbf{L 1}, \mathbf{U 1})$, stating that $L 1$ and $U 1$ are the minimum and maximum delays between $P 1$ and $P 2(L 1 \leq U 1)$, where $P 1$ and $P 2$ are time points (i.e., starting/ending points of events). As regards qualitative temporal constraints (e.g., before, during), ITL considers all and only the qualitative constraints that can be mapped onto conjunctions of STP constraints (this subset proved to be very important in many practical applications (VanBeek 1991, Brusoni et al. 1997, Console and Terenziani 1999)).

The semantics of ITL constraints is specified in terms of STP constraints as follows:

$$
\begin{aligned}
& \operatorname{date}(\mathbf{E}, \mathrm{L} 1, \mathrm{U} 1, \mathrm{~L} 2, \mathrm{U} 2) \Leftrightarrow\left(\mathrm{L} 1 \leq \mathrm{Start}(\mathrm{E})-\mathrm{X}_{0} \leq \mathrm{U} 1\right) \wedge\left(\mathrm{L} 2 \leq \mathrm{End}(\mathrm{E})-\mathrm{X}_{0} \leq \mathrm{U} 2\right) \\
& \operatorname{duration}(\mathbf{E}, \mathrm{L1}, \mathrm{U1}) \Leftrightarrow \mathrm{L} 1 \leq \mathrm{End}(\mathrm{E})-\mathrm{Start}(\mathrm{E}) \leq \mathrm{U} 1 \\
& \operatorname{delay}(\mathbf{P 1}, \mathbf{P} 2, \mathrm{L1} 1, \mathrm{U} 1) \Leftrightarrow \mathrm{L} 1 \leq \mathrm{P} 2-\mathrm{P} 1 \leq \mathrm{U} 1
\end{aligned}
$$

Notice that dates are represented by distances from a chosen time point $X_{0}$, which is unique for the whole knowledge base. As examples of qualitative relations, let us consider before and during between two time intervals $E 1$ and $E 2$ :

$$
\begin{aligned}
& \text { before }(\mathrm{E} 1, \mathrm{E} 2) \Leftrightarrow 0<\operatorname{Start}(\mathrm{E} 2)-\mathrm{End}(\mathrm{E} 1) \\
& \text { during(E1,E2) } \Leftrightarrow(0<\operatorname{Start}(\mathrm{E} 1)-\operatorname{Start}(\mathrm{E} 2)) \wedge(0<\operatorname{End}(\mathrm{E} 2)-\operatorname{End}(\mathrm{E} 1))
\end{aligned}
$$

\footnotetext{
* Notice, however, that arbitrary disjunctions of temporal constraints (such as in 'LT1 was during LT2 or LT2 lasted at least 1 hour') cannot be specified by conjunctions of STP constraints, as well as some disjunctive relations in Allen's algebra such as 'LT1 before or after LT2', or relative durations such as 'LT1 lasted twice than LT2'.
} 


\subsubsection{Other constructs of ITL}

The temporal constraint language described above is very similar to the languages employed by many temporal managers in AI literature (see, e.g., (Dechter et al. 1991, Meiri 1991, Vila 1994, Brusoni et al. 1997)). In order to be able to integrate temporal constraints between classes and between instances, we must extend ITL. We introduce the primitive Instance_of(E1,C1) to state that $E 1$ is a specific instance of the class of events $C 1$ (for instance, in figure 1, we have Instance_of(R1,Res), Instance_of(R2, Res), etc.).

The primitive COR is introduced to represent correlations between instances of events.

Example. For instance, in figure 1, we assume that $R 1$ is correlated with $T 1$ (via the correlation relation $C_{1}$ ) and $R 2$ is correlated to $T 2$ (via the correlation relation $C_{2}$ ). This can be expressed in ITL by:

$\mathrm{COR}\left(\mathrm{C}_{1}, \mathrm{R} 1, \mathrm{~T} 1\right), \mathrm{COR}\left(\mathrm{C}_{2}, \mathrm{R} 2, \mathrm{~T} 2\right)$.

Finally, it is useful to indicate explicitly the set IKB_Elements of all the instances (e.g., $\{R 1, R 2, T 1, T 2\}$ in figure 1$)$.

\subsubsection{Knowledge base of instances}

In ITL, a Knowledge Base about instances of events (IKB for short) is a quadruple $<I K B \_$Elements, IKB_Instance_of, IKB_COR, IKB_Constraints $>$, where IKB_Elements is a set of instances of events, IKB_Instance_of is a set of Instance_of assertions, IKB_COR a set of correlations and $I K B$ _Constraints a set of (i.e., a conjunction of) temporal constraints on instances of events.

While the semantics of temporal constraints in our language has been simply expressed in terms of STP constraints, the implicit temporal meaning of having a given instance $i$ in the IKB_Elements component of IKB depends on the assumptions one makes regarding the possibility of dealing with future instances of events in IKB and on whether IKB is complete or not. In the following, we introduce such issues mainly through first-order formulae, which thus can be seen as $\underline{a}$ declarative specification of some of the inferences that the classes+instances temporal reasoning algorithms have to perform ${ }^{\dagger}$.

(1) (Ax1) covers the cases where the IKB is a representation of the instances of events which have been observed until RT (i.e., future instances of events cannot be inserted in IKB_Elements):

$$
\text { (Ax1) } \forall \mathrm{x} x \in \mathrm{IKB} \text { Elements } \Rightarrow \operatorname{Start}(\mathrm{x})-\mathrm{RT} \leq 0
$$

(Ax1) states that if an instance $x$ of event has been observed (i.e., $x \in I K B$ Elements), then it has been observed to start before (or equal to) RT (otherwise, if also future instances are taken into account in IKB, the fact that $x \in I K B \_$Elements does not provide any implicit indication on the temporal location of $x$ ).

(2) In case we assume that observations are complete, the fact that an instance $x$ of event has not been observed (i.e., $\left.x \notin I K B \_E l e m e n t s\right)$ has different temporal implications, depending on whether the system takes into account future events or not. With complete observations and no future events, each instance that does not belong to IKB_Elements has not occurred yet (i.e., did not start before RT; this can be formalized as in $\mathrm{Ax} \overline{2}$ ).

$$
(\mathrm{Ax} 2) \forall \mathrm{x} \mathrm{x} \notin \mathrm{IKB} \_ \text {Elements } \Rightarrow \mathrm{NOT}(\operatorname{Start}(\mathrm{x})-\mathrm{RT} \leq 0)
$$

On the other hand, with complete observations and future events, the fact that an event instance does not belong to IKB_Elements implies that it never occurs in the past, present and future.

† As in Allen's seminal approach (Allen 1983, 1984, Allen and Hayes 1989), we use logical formulae to specify formally the intended meaning of our constraint language, and we define specialised temporal reasoning techniques to operate on temporal constraints in an efficient way, respecting the logical semantics of the constraints. In fact, specialised reasoners are usually more efficient that a general purpose theorem prover for a logic (Allen 1983, 1984, Allen and Hayes 1989). 


\subsection{Language for temporal constraints between classes of events (CTL)}

\subsubsection{Temporal constraint language for classes}

In general, all the types of temporal constraints discussed above can also be expressed between classes of events. Thus, we use the same constraint language as above (however, ' $\mathrm{C}$ ' is used as a prefix to indicate predicates applying to classes) to express them into our temporal language for classes of events ( $\underline{\mathrm{CTL}}$ for short). However, the semantics of temporal constraints on classes is different. In fact, when applied to classes, dates, durations, delays (here we consider just the delays between the starting points of two classes; the other cases are analogous) and qualitative relations have a different meaning. This meaning can be expressed, for instance, as shown below."

Cdate(C,L1,U1,L2,U2) $\Leftrightarrow \forall \mathrm{C}^{\prime}$ (Instance_of(C',C) $\Rightarrow\left(\left(\mathrm{L} 1 \leq \operatorname{Start}\left(\mathrm{C}^{\prime}\right)-\mathrm{X}_{0} \leq \mathrm{U1}\right) \wedge(\mathrm{L} 2 \leq\right.$ $\left.\left.\operatorname{End}\left(\mathrm{C}^{\prime}\right)-\mathrm{X}_{0} \leq \mathrm{U} 2\right)\right)$

Cduration(C,L1,U1) $\Leftrightarrow \forall \mathrm{C}^{\prime}\left(\right.$ Instance_of $\left.\left(\mathrm{C}^{\prime}, \mathrm{C}\right) \Rightarrow \mathrm{L} 1 \leq \operatorname{End}\left(\mathrm{C}^{\prime}\right)-\operatorname{Start}\left(\mathrm{C}^{\prime}\right) \leq \mathrm{U} 1\right)$

$\operatorname{Cdelay}(\operatorname{Start}(\mathrm{C} 1), \operatorname{Start}(\mathrm{C} 2), \mathbf{L} 1, \mathrm{U} 1) \quad \Leftrightarrow \quad\left(\forall \quad \mathrm{C} 1^{\prime}, \mathrm{C} 2{ }^{\prime}, \mathrm{Cor} \quad\right.$ (Instance_of(C1',C1) $\wedge$ Instance_of $\left(\mathrm{C} 2{ }^{\prime}, \mathrm{C} 2\right) \wedge$

$\left.\left.\mathrm{COR}\left(\mathrm{Cor}, \mathrm{C} 1^{\prime}, \mathrm{C} 2^{\prime}\right)\right) \Rightarrow\left(\mathrm{L} 1 \leq \operatorname{Start}\left(\mathrm{C} 2^{\prime}\right)-\operatorname{Start}\left(\mathrm{C} 1^{\prime}\right) \leq \mathrm{U} 1\right)\right) \wedge$

$\left(\forall \quad \mathrm{C} 1^{\prime} \quad\right.$ Instance_of $\left(\mathrm{C} 1^{\prime}, \mathrm{C} 1\right) \Rightarrow \quad\left(\exists \quad \mathrm{C} 2^{\prime}, \mathrm{Cor} \quad\right.$ Instance_of $\left(\mathrm{C} 2^{\prime}, \mathrm{C} 2\right) \wedge$

$\left.\left.\mathrm{COR}\left(\mathrm{Cor}, \mathrm{C} 1^{\prime}, \mathrm{C} 2 '\right)\right)\right)$ )

As an example of qualitative relations, let us consider the relation 'before':

Cbefore(C1,C2) $\Leftrightarrow\left(\forall\right.$ C1',C2',Cor (Instance_of(C1',C1) $\wedge$ Instance_of $\left(C 2{ }^{\prime}, C 2\right) \wedge$ $\left.\mathrm{COR}\left(\mathrm{Cor}, \mathrm{C} 1^{\prime}, \mathrm{C} 2^{\prime}\right)\right) \Rightarrow\left(0<\operatorname{Start}\left(\mathrm{C} 2^{\prime}\right)-\operatorname{End}\left(\mathrm{C} 1^{\prime}\right)\right) \wedge\left(\forall\right.$ C1' Instance_of $\left(\mathrm{C} 1^{\prime}, \mathrm{C} 1\right) \Rightarrow$ $\left(\exists \mathrm{C} 2\right.$ ',Cor Instance_of $\left.\left.\left.\left(\mathrm{C} 2{ }^{\prime}, \mathrm{C} 2\right) \wedge \mathrm{COR}\left(\mathrm{Cor}, \mathrm{C} 1^{\prime}, \mathrm{C} 2{ }^{\prime}\right)\right)\right)\right)$

While durations are simply inherited by all instances, qualitative relations and delays are only inherited by correlated pairs of instances (see section 2.1). The second conjuncts in the definition of Cdelay and Cbefore formalize the 'predictive' character of delays and qualitative relations between classes of events. For example, given the constraint between classes Cbefore $(C 1, C 2)$, the observation of an instance of $C 1$ implies the subsequent occurrence of a correlated instance of $C 2$. Moreover, as required by many practical application areas, CTL also copes with temporal constraints on the repetition of events and on their periodicity. In our approach (which is also based on the results of the linguistic analysis by Van Eynde (1987)), we deal with different components in the definitions of repeated actions, in order to deal also with complex cases such as Ex. 1 and Ex. 5:

(Ex. 5) For six months, perform action A twice each five days for twenty days and then suspend for ten days (and so on).

Thus we can deal with frame times ('the interval which contain all the instances of the event' (Van Eynde 1987), e.g., 'for six months' in Ex. 5 - henceforth called FT for short). The description of repeated periodic events splits frame times into a sequence of intervals when actions are performed (called action-times - AT for short; e.g. 'twenty days' in Ex. 5) and 'pause' intervals (delay time - DT for short - e.g., 'ten days' in Ex. 5). In turn, action times are split into I-times (IT for short; e.g., 'five days' in Ex. 5) where actions are performed (if DT is null, $A T$ coincides with IT). Finally, we call the number of actions in each I-time 'frequency' (freq for short; e.g., 'twice' in Ex. 5). The above terms are illustrated in figure 3.

\section{insert figure 3 about here.}

Notice that, as in many AI approaches in the literature (see, e.g., (Nebel 1990)), we specify the semantics of classes in an extensional way, i.e., considering classes as sets of instances. 
Thus, we introduce in our constraint language the primitive

\section{Repetition_Periodicity(FT,AT,DT,IT,freq,C)}

to state the constraints on the repetition of the (class of) event $C$.

Notice that, since we aim at designing tractable algorithms to deal with correct and complete consistency checking, we have to impose the constraint that FT, AT, DT, IT and freq must be specified in an exact way.

The logical semantics of the predicate Repetition_Periodicity is quite straightforward and is shown in the Appendix A.

Finally, in our language, we use the primitive

\section{Part_Of(C1,C)}

to state that a class $C 1$ is part of a class $C$. We will see in the rest of the paper how we cope with the temporal constraints between the composite and the component classes of events induced by the Part_Of relation.

Thus, in our language, a knowledge base CKB of classes is a triple $<$ CKB_Elements, $C K B \_P a r t \_O f, C K B \_C o n s t r a i n t s>$, where the first term represents the set of classes. ${ }^{\S}$

\subsection{Internal representation for temporal constraints: the STP-tree}

The high-level constraint language about instances has been deliberately designed in such a way that all constraints can be easily mapped onto bounds on differences and, thus, internally represented as a 'standard' STP framework (Dechter et al. 1991).

The same point also holds for dates, delays, durations and qualitative temporal constraints between classes of events, that might be stored into a separate STP dealing with the constraints about classes (where each class is represented by a starting and an ending point) ${ }^{* *}$. However, such a basic approach must be extended to cope with (the temporal constraints involved by) part-of relations and with the temporal constraints about repeated events .

As regards part-of relations, we represent the temporal constraints they involve as constraints between the starting/ending points of the composite event and the endpoints of its (direct) subactions. In particular, the start of a composite event is temporally equal to the start of its first sub-event, while its end is equal to the end of its last sub-event ${ }^{\dagger \dagger}$. Therefore, the constraints

\footnotetext{
$\S$ Notice that, in this representation, we assume that the temporal constraints in CKB_Constraints implicitly represent the possible presence of a correlation relation between the corresponding instances. In other words, given two instances $E 1$ and $E 2$ of two concepts $C 1$ and $C 2$ respectively, $E 1$ and $E 2$ may be correlated only in case $C 1$ and $C 2$ are related via some temporal relation in the CKB (notice that the relation $C 1 A L L C 2$ can be used in order to relate $C 1$ and $C 2$ stating that any temporal constraint can hold between them, i.e., that $-\infty \leq S_{C 1}-S_{C 2} \leq+\infty \wedge-\infty \leq S_{C 1}-E_{C 2} \leq+\infty$ $\left.\wedge-\infty \leq E_{C 1}-S_{C 2} \leq+\infty \wedge-\infty \leq E_{C 1}-E_{C 2} \leq+\infty\right)$. In other words, we assume (Ax3):

(Ax3) $\forall \mathrm{c} 1 \in \mathrm{CKB}$ EventClass, $\forall \mathrm{c} 2 \in \mathrm{CKB}$ EventClass $\quad$ NOT $\quad($ Connected(CKB,c1,c2) $\Rightarrow$ $\left(\forall \mathrm{e} 1, \mathrm{e} 2\right.$ Instance_of $(\mathrm{e} 1, \mathrm{c} 1) \wedge$ Instance_of(e2,c2) $\Rightarrow$ NOT $\exists$ Cor $\left.\left._{\mathrm{i}}\left(\mathrm{COR}\left(\mathrm{Cor}_{\mathrm{i}}, \mathrm{e} 1, \mathrm{e} 2\right)\right)\right)\right)$

where Connected $(c 1, c 2)$ informally represents the fact that the classes of events $c 1$ and $c 2$ are connected vertices in the graph formed by the constraints in $C K B$ Constraints.

${ }^{* *}$ Two different STPs are needed in order to model the fact that, basically, constraints between instances are 'existentially quantified', while constraints between classes are 'universally quantified' (Terenziani 2002).

it Unfortunately, if the temporal constraints between the sub-events are such that one cannot find its first and last sub-events, the above constraint involves a disjunction, and cannot be modelled in the STP framework (for instance, an approach such as (Galipienso and Barber Sanchís 2002) would be needed). On the other hand, in the STP framework, one can
} 
involving classes of events (which are not repeated) can be homogeneously represented in a unique STP framework, containing a starting and an ending point for each composite and atomic event ${ }^{*}$.

However, such a homogeneous approach does not work in the case of repeated/periodic events. In fact, in many real-world application domains (e.g., medical therapies), actions can be repeated a relevant number of times, so that an explicit representation of all repetitions could cause an unnecessary explosion of the number of points in the STP ${ }^{\S \S}$. Such an explosion can be avoided via an 'intensional' representation, in which a single entity (e.g., a time interval, possibly represented via its endpoints) is used to represent the set of all repetitions of a given event. However, such an intensional approach cannot be directly implemented in 'classical' temporal constraint propagation approaches (such as, e.g., STP), which need an explicit (i.e., 'extensional') representation of all the temporal entities (time points and/or time intervals). For instance, the temporal constraint that the end of a repeated event $E_{1}$ is 1 hour before the starting point of a repeated event $E_{2}$ is actually a constraint between the end of the last repetition of $E_{1}$ and the first repetition of $E_{2}$. Furthermore, the components of the specification of the periodicity of repeated events (e.g., $A T, D T, I T)$ are actually constraints on the single repetitions (rather than on the repeated event as a whole). But, in the intensional approach, one does not want an explicit representation of each single repetition...

We thus chose to model repeated events as composite events and to represent the constraints regarding repeated actions into separate STPs, one for each repeated event. Thus, in our approach, the overall set of constraints between classes of events is represented by a tree of STPs (STP-tree henceforth) (Terenziani et al. 2002b). The root of the tree is the STP which homogeneously represents the constraints between all the classes of events (composite and atomic), except repeated events. Each node in the tree is an STP and has as many children as the number of repeated events it contains. Each edge in the tree connects a pair of endpoints in an STP (the starting and ending point of a repeated event) to the STP containing the constraints between its subactions and is labelled with a list of properties describing the temporal constraints on the repetitions (i.e., $F T, A T, D T, I T$, freq). For example, in figure 4, we show the STP-tree representing temporal constraints involved by the example Ex. 1 in Section 2.

\section{insert figure 4 about here.}

\section{Temporal reasoning on classes}

As described above, each node of the STP-tree contains an STP, and the edges linking the nodes carry information about the repetition/periodicity. Thus, in order to check the consistency of the

represent the weaker constraint that all sub-events start after or at the same time than the start of the event they compose, and end before or at the same time of its end.

\# Alternatively, we could have chosen to partition the constraints about classes into a set of STP frameworks, one for each composite event. Such a clustering technique would save space and, apparently, make the reasoning process more efficient. However, the temporal constraints between the composite events and their components make the constraints in different STPs dependent on each other. Thus, to determine the global consistency of such a set of STPs one would need to perform a potentially never-ending iteration of the reasoning process, until a state of quiescence is reached.

$\S \S$ Such a representation of each repetition would also make the problem of maintaining the coherence of data very expensive, in the case of updates of the knowledge base of constraints. 
STP-tree, it is not sufficient to check the consistency of each node separately by means of the well-known all-pairs shortest paths algorithms that solve the problem for STPs (Dechter et al. 1991). In this case, in fact, we would neglect the repetition/periodicity information. Temporal consistency checking thus proceeds in a top-down fashion, starting from the root of the STP-tree. Basically, the root contains a 'standard' STP, so that the Floyd-Warshall's algorithm can be applied to check its consistency. Thereafter, we proceed top down towards the leaves of the tree. For each node $X$ in the STP-tree (except the root), we progress as shown in the algorithm STP_tree_consistency in figure 5 .

\section{insert figure 5 about here.}

STP_tree_consistency accepts as input the STP-node that must be checked and the constraint about the repetition (i.e., the constraint in the arc of the STP-tree entering node $X$; namely, the duration of the FT, of the AT, of the IT, of the DT and a frequency), and gives as an output an inconsistency, or, in case of consistency, the local minimal network of the constraints in $X$ plus those about the repetition.

In step 1 we check whether the repetition constraint is 'well-formed', i.e. if it is consistent when it is taken in isolation (i.e., if $A T+D T$ is contained in $F T$, and $I T$ is contained in $A T$ ).

In step 2 we propagate the constraints in $X$ in order to obtain the minimal network $Y$, which is useful for having the minimum and maximum distances between the points in the STP. In steps 49 we make sure that all the specified (freq) repetitions in an IT can be performed. In particular, in steps 4-5 we compute the maximum distance between any points in the STP that still allows to perform all the required repetitions. This is obtained by considering the distance (step 5) that allows to perform a repetition assuming that all the other repetitions in the same IT have the minimum possible duration. In steps 6-7 the constraints are added to the STP and, finally, in step 8 we check their consistency.

The algorithm takes advantage also of a conversion table mapping each granularity provided to the user (currently, we provide a fixed menu of granularities, not admitting user-defined granularities (Bettini et al. 2002, Combi et al. 2004)) into the basic granularity adopted by the reasoning process.

Complexity. The complexity of the algorithm is dominated by steps 2 and 8 , that are $\mathrm{O}\left(C^{3}\right)$, where $C$ is the number of classes in the CKB.

Property 1. The top-down visit of the STP-tree is complete as regards consistency checking of the constraints in the STP-tree.

Proof (sketch). The all-shortest-paths algorithm is complete for the STP framework. The only constraints relating actions (or, better, time points) in different STPs in the tree are located on the edges of the tree, and, by definition, the STP-tree does not allow loops between STPs. Since FT, $A T, I T, D T$ and freq (if any) must be provided in a precise (exact) way, they cannot be further 'restricted' by constraint propagation. Thus, there is no need to propagate forward and backward the constraints along the tree, and a top-down visit of the tree is sufficient ${ }^{* * *}$.

\footnotetext{
*** Notice, however, that the above reasoning mechanism does not provide the minimal network between all the actions.
} 


\section{Integrated classes+instances temporal reasoning}

\subsection{Integrated classes+instances temporal reasoning with complete knowledge}

In this section, we describe two temporal reasoning algorithms to check the consistency of a knowledge base of temporal constraints regarding both classes and instances of events. For the sake of clarity, we adopt an incremental presentation: first, we describe our basic algorithm, which assumes full observability of instances even in the future and total ordering between instances of events. Next, we extend such an algorithm in order to work in domains where there is no observability in the future. The problem of relaxing the assumptions of total ordering and full observability in the past is briefly investigated in section 6 , and is one of the main goals of our future work.

The algorithm in figure 6 works in three steps. The procedure integratedConsistency:

- calls the procedure unfoldClasses in order to make explicit the constraints intensionally implied by the periodicity/repetition constraints;

- inherits the constraints onto the instances;

- propagates the constraints about the instances.

The arguments of procedure integratedConsistency are the high-level description of the classes and the high-level description of the instances (respectively expressed by the knowledge bases of classes (CKB) and instances (IKB), see section 3). For the sake of brevity, we assume that all the input classes and instances are correlated. It should be pointed out that this is not restrictive: in fact, since the correlation relation partitions actions (and their constraints) into independent sets (Morris et al. 1993, Terenziani 1997), the algorithms can be iterated on each set.

\section{insert figure 6 about here.}

The procedures unfoldClasses, unfoldSTPNode and unfoldRep in figure 6 are meant to fill the gap between the high-level intensional representation language and the low-level extensional internal representation as STP ((Dechter et al. 1991)), making explicit the semantic assumptions carried by the intensional high-level language. At the end of step 3 of the procedure unfoldClasses, $S$ is a 'flat' STP which is semantically equivalent to the STP-tree $T$.

This task is accomplished by:

- visiting recursively the STP-tree (task performed by the procedure unfoldSTPNode);

- 'unfolding' (i.e., making explicit) the repetitions (task performed by the procedure unfoldRep).

Before each recursive call (i.e., in step 2 of unfoldClasses, step 5 of unfoldSTPNode and step 7 of unfoldRep), a 'placeholder' class is inserted into the STP. This dummy class is useful in order to represent the abstract classes that stand for the entire set of repetitions that will be unfolded. Note that the placeholder class $\mathrm{C}_{\mathrm{T}}$ added in unfoldClasses and representing the root node of the STPtree - actually not necessary - is added for the sake of uniformity.

The procedure unfoldSTPNode is recursively called on each STP-node $X$ in the STP-tree. It copies in $S$ all the atomic, non repeated classes (step 2), imposing the constraint that they are contained in the placeholder class $C_{X}$ (step 3).

For each repeated class (steps 4-8), it calls the procedure unfoldRep in order to 'unfold' the repetitions. Finally, in steps 9-10 the monadic and binary temporal constraints in $X$ are copied to $S$, relating them to the corresponding copied classes. It should be pointed out that, while for atomic classes the algorithm simply copies the constraints from $X$ to the copied atomic classes in $S$, for non-atomic classes the algorithm copies the constraints to the related placeholder classes added to $S$ (i.e., on the $F T_{C r}$ placeholder classes). 
In the procedure unfoldRep, the three nested for loops in steps 1, 4 and 6 accomplish the task of 'unfolding' the repetitions, i.e., the actions must be executed as many times as the class $C$ is repeated. Before each for loop (steps 2, 3, 5, 7), a placeholder class is inserted into the STP in order to represent the entire set of repetitions. In step 8 the procedure unfoldSTPNode is called in order to continue the unfolding on the STP-node child of $X$ in the STP-tree.

Finally, unfoldRep adds to the STP (steps 9-12) the constraints corresponding to the logical semantics of the repetition constraint on $C$ (see Appendix A for more details): they have a fixed duration (step 9), they are mutually contained (i.e., freq $q_{i j k} \subseteq I T_{i j} \subseteq A T_{i} \subseteq A T D T_{i} \subseteq F T_{C}$ and $D T_{i} \subseteq$ $A T D T_{i}$ ) (step 10), each placeholder in the above containment chain starts the containing placeholder (e.g., freq $q_{i j 1}$ starts $I T_{i j}$; step 11) and the placeholders meet (step 12).

As regards the procedure integratedConsistency, as above-stated, after the call to unfoldClasses, it has in $S$ an STP semantically equivalent to the CKB $T$. The rest of the procedure is devoted to:

- finding a one-to-one correspondence among the temporal points in $\mathrm{S}$ that describe the starting and ending points of the 'unfolded' classes, and the temporal points in $I$, that describe the starting and ending points of the instances of events (steps 2-8);

- inheriting the temporal constraints from classes onto the instances (steps 9-10);

- checking the consistency of the instances (steps 11-12).

As observed in section 2.3, the knowledge base of instances usually does not contain the instances of composite events, that are therefore added to the instances (steps 2 and 3 ) in order to make it easier to relate classes to the corresponding instances. In particular, steps 1 and 2 make explicit the repetitions by:

(i) generating and instantiating the placeholders for the periodic pattern (i.e., $F T_{i} \mathrm{~s}, A T_{i} \mathrm{~s}, D T_{i} \mathrm{~s}$, $I T_{i} \mathrm{~s}$, freq $\left._{i} \mathrm{~s}\right)$; and

(ii) generating -in sequence- a class for each repetition of a repeated action, locating it properly within the periodic pattern.

In such a context, the problem of associating instances to periodic patterns (see section 2.2) can be solved by finding a correspondence between the instances in IKB and the unfolded classes obtained in (ii). In the procedure integratedConsistency we rely on the assumption that instances are ordered in order to find such a correspondence. More specifically, step 4 propagates the constraints in order to infer a possibly implicit temporal ordering between instances, and the for loop in step 6 takes into account one class at a time considering the lexicographic order given by the pair (starting time, ending time) of the class. Then, step 7 finds the corresponding instance, considering both the instance-of and the temporal ordering information. If an instance is missing (step 8) or if there are more instances than unfolded classes (step 9), we report an error because there is a mismatch between the number of actions specified at the class level and the number of instances. Notice that, since here we assume full observability also in the future and we consider only correlated classes and instances, each class must have exactly one instance, and vice versa.

Finally, after inheriting the temporal constraints (steps 10-11), the augmented STP is checked for consistency of the whole set of constraints (steps 12-13).

Optimization 1. The algorithms in figure 6 perform the 'unfolding' of repeated actions in a quite 'naïve' way, in the sense that all the possible repetitions of classes are made explicit. However, the unfolding of classes is actually performed as a first step in the process of inheriting constraints from classes to instances (of course, instances are naturally 'unfolded'). This means that there is no need to unfold the repetitions of classes which have no related instances. Thus, the algorithms can be optimized by stopping the unfolding and (in the case of full observability even in the future) reporting an inconsistency as soon as a class has not a corresponding instance.

Complexity. In order to compute the complexity of the algorithm, it is useful to observe that unfoldClasses, unfoldSTPNode and unfoldRep procedures, thanks to their recursive structures and to the nested for loops, basically traverse the STP-tree, visiting each STP-node as many times as 
the number of times it must be repeated. Therefore, it is handful to express the complexity of the algorithm in terms of the number $L$ of the unfolded classes, rather than the number of input classes in the CKB (if we denote with $C$ the number of the input CKB classes, and with $R$ the maximum number of times that any class is repeated in the STP-tree, we can estimate $L$ as $\mathrm{O}(R * C))$. As regards the procedure unfoldRep, the three nested for statements (steps 1, 4 and 6), together with the inner mutually recursive call in step 8 , simply 'unfold' all the repetitions of classes, and the overall complexity is thus linear in $L$. Therefore, the complexity of procedure unfoldRep is dominated by steps 9-11 (adding constraints on the repetitions), which are linear in the number of unfolded classes. Analogously, the complexity of procedure unfoldSTPNode is dominated by step 10 (adding the binary constraints), which is quadratic in the number of unfolded classes, and, finally, procedure unfoldClasses is dominated by step 4 (performing constraint propagation), which is cubic on the number of the unfolded classes.

Therefore, step 1 of procedure integratedConsistecy is $\mathrm{O}\left(L^{3}\right)$. Thanks to a possible precompilation (performable in $\mathrm{O}(S)$, where $S$ is the number of instances) that associates with each class its instances, and thanks to the total ordering of the instances, step 7 can be performed in constant time and the entire for loop in steps 6-8 is linear in the number of classes. As regards the inheritance of the constraints in steps 10-11, its complexity is dominated by the inheritance of binary constraints, which is quadratic in the number of classes.

Therefore, in the procedure integratedConsistency, steps 1, 4 and 12 dominate, and the overall complexity is $\mathrm{O}\left(\max \left\{L^{3}, S^{3}\right\}\right)$. Notice that, in case Optimization 1 is adopted, $L \leq S$. Thus, in such a context, the overall complexity is $\mathrm{O}\left(S^{3}\right)$, which is the complexity of applying Floyd-Warshall to the STP of instances. Therefore, the unfolding and the inheritance parts of the algorithm do not affect the asymptotical complexity.

Conjecture. It can also be conjectured that the temporal reasoning algorithms in figure 6 are complete. In fact, the unfolding procedure makes all the repetitions of classes explicit, and inheritance procedures add on the proper instances all the bounds-on-differences mappings of the temporal constraints between classes (according to the logical semantics we specified in sections 3.2 and 3.3, and in Appendix A). Finally, complete temporal reasoning on instances is performed via Floyd-Warshall's algorithm (which is complete for STP problems (Dechter et al. 1991)).

\subsection{Integrated temporal reasoning with no future events}

In this section we relax the assumption of full observability and we assume that we have no instances of events in the future.

\section{insert figure 7 about here.}

The procedure integratedConsistencyNoFuture in figure 7 extends the procedure integrated Consistency presented in the previous section along such a direction. The steps added or changed wrt the procedure integratedConsistency are the ones in bold and underlined type. The other parts of the algorithm don't change.

integratedConsistencyNoFuture accepts the additional parameter RT, corresponding to the time of the present. In steps 1a-1b we explicit the assumption that it is not possible to observe future events: all observed instances must start before RT (see Ax1 in section 3.2.3).

However, the main differences between the procedures integratedConsistencyNoFuture and integratedConsistency lie in steps 7-7b and 13: when we do not find an instance that a class predicts to be in the IKB, we no longer report an inconsistency, because that instance may start in the future. Thus, there is an inconsistency only in the case that the temporal constraints in IKB and 
CKB impose that the instance must be observed before RT (see Ax2 in section 3.2.3). Therefore, we collect all the missing instances in the set hypothesizedInstances (steps 7 and 7a) and we provisionally insert them in IKB (step 7b). Then, we perform the inheritance and the propagation of the constraints on input+hypothesized instances, and (step 13) we test whether any hypothesized instance necessarily starts before RT. In this case we report the inconsistency, because we should have been able to observe it before RT, but we couldn't.

Optimization 2. It is worth noting that, in the case that the missing instances belong to a repeated class, it is not necessary to hypothesize all the repetitions, but only the first missing one; in fact, if this instance may start in the future, also the subsequent ones will, and it is not necessary to hypothesize them; on the other hand, if this instance must start before RT, we may report the inconsistency even without hypothesizing the others. Similarly, it is not necessary to unfold all the classes, but only the ones that correspond to instances actually present in the IKB (see Optimization 1).

Complexity. In the procedure integratedConsistencyNoFuture, steps 1a-1c are linear in the number of instances, and steps 7-7b are linear on the number of classes. It is worth noting that for step 13 we may exploit the locality properties of STP constraints proved in (Brusoni et al. 1995) and perform it in a time linear in the number of instances in hypothesizedInstances. The cardinality of hypothesizedInstances is at most $L$, so that the overall complexity is $\mathrm{O}\left((L+\mathrm{S})^{3}\right)$. Notice, however that, in case Optimization 2 is adopted, we at most hypothesize one instance for each input (i.e., not unfolded) class. As above, let $C$ be the number of such classes. Thus the overall complexity becomes $\mathrm{O}\left((C+S)^{3}\right)$.

\subsection{Preliminary experimental results}

The theoretical approach described in this paper has been implemented in TEMPORE, a prototype of domain-independent temporal reasoner. TEMPORE has been implemented in Java (JDK 1.4). The experiments were run on a PC with a Pentium IV CPU at $2 \mathrm{GHz}$ with $1 \mathrm{~GB}$ RAM and Windows 2000 operating system.

In the following, we sketch our initial experiments (we plan to perform further experiments concerning also the algorithms in figure 7).

\section{insert table 1 about here}

The prototype was provided with six different pairs of class and instance knowledge bases, with increasing number of actions. In table 1 the number of unfolded classes of the knowledge bases and the relative CPU times for consistency checking are reported. The experiments regard the algorithms in figure 6, i.e., the cases where full observability and future events are considered. In such cases, (in the case of consistency) the number of instances is equal to the number of unfolded classes. Notice that, since in the STP encoding each class/instance is represented by its starting and its ending point, the actual number of entities on which the propagation algorithms operate is twice the number of classes specified in the table.

\section{Conclusions and developments}

In this paper, we describe a tractable temporal manager (TEMPORE) coping in an integrated way with different temporal aspects involved in real-world temporal applications. In particular, TEMPORE offers an expressive high-level language for explicitly representing part-of and instance-of relations between events, as well as (a limited set of qualitative and quantitative) 
temporal constraints between classes and instances of events, including periodic repetitions. Moreover, it supports temporal reasoning for consistency checking. Despite the increased expressiveness, however, temporal reasoning in TEMPORE is still tractable, and the resulting asymptotical complexity is very close (in the case of no future events) or equal (in the case of future events) to the complexity of performing consistency checking (e.g., via the FloydWarshall's algorithm) on an STP of instances only.

\subsection{Applications}

The requirement of coping with the temporal constraints rising from so many different aspects of reality (ranging from part-of and instance-of relations to standard qualitative and quantitative temporal constraints and to periodicity) emerged from our previous work in different application fields (including train scheduling (Brusoni et al. 1996) and multimedia presentation (Adali et al. 2000)), and, in particular, in clinical guideline management. Since 1997, we work in a joint project with Azienda Ospedaliera S. Giovanni Battista of Turin (one of the main hospitals in Italy) for the design and the development of GLARE (GuideLine Acquisition, Representation and Execution) (Terenziani et al. 2001, 2002a, b), a system which acquires and represents clinical guidelines, and then executes them (in a semi-automatic way) on specific patients.

In such an application, all the expressiveness of TEMPORE is needed. In fact, clinical guidelines describe general procedures (i.e., procedures about classes of events), and are structured via partof relations. Many actions in the guidelines (especially in the therapeutic part of guidelines) must be repeated periodically. Temporal constraints (e.g., delays) are used in order to constrain the execution of actions in the guidelines. Moreover, clinical guidelines are executed on specific patients, so that one has to represent and store the execution times. In particular, notice that full observability in the past and total ordering on correlated instances are reasonable assumptions considering the execution of clinical guidelines on hospitalized patients. Specifically, in our application domain, instances are exactly located in time.

TEMPORE has been loosely coupled with GLARE in order to operate as a temporal-constraint manager, dealing with the problem of representing and reasoning with temporal constraints in the guidelines. Specifically, the temporal reasoning facilities provided by TEMPORE are exploited in different ways in GLARE, providing crucial advantages both in the acquisition and in the execution phase (Terenziani et al. 2003).

During acquisition, expert physicians (usually with the help of a knowledge engineer) have to represent in a computer-based formalism a clinical guideline, including the temporal constraints it contains. Checking the consistency of such constraints is a fundamental task, which cannot be performed directly by expert physicians (and/or knowledge engineers). Thus, an automated consistency-checking facility is very important in this phase, to alleviate the physicians' work and to grant the (temporal) consistency of the guideline in a principled way. In passing, notice that the temporal reasoning facility can be advocated at any stage during the acquisition of a clinical guideline, so that incremental consistency checking is also possible (e.g., at the end of each working session, or whenever a 'significant' portion of guideline has been added).

GLARE allows users to execute acquired guidelines on specific patients, automatically gathering the patients' data from the patient database. Also during execution, temporal reasoning facilities can provide important advantages.

First of all, a guideline describes a set of alternative procedures to deal with a given disease. By its own nature, it describes 'general' procedures, to be instantiated on specific patients. This is particularly true as concerns the temporal constraints in a guideline: They provide general 'temporal patterns' which must be 'respected' by each specific execution (instantiation) of the guideline. Thus, (a) temporal reasoning can be advocated 'a priori' during execution, to provide information regarding the temporal frame in which the next action has to be performed, given the constraints in the whole guideline and given the time when the last action in the guideline has 
been executed. From the point of view of quality evaluation/assessment, also an 'a posteriori' use can provide significant advantages: (b) Temporal reasoning can be activated after the execution of (part of) a guideline, in order to check whether the temporal constraints in the guideline have been respected (until now) or not.

Second, one of the main goals of guideline computer-based systems is to support decision-making. In general, current computer-based approaches to clinical guidelines provide to the user-physician only the pieces of information which constitute the description of the decision at hand. In some cases, however, additional information, gathered from the paths in the guideline that follow the decision at hand, can be important in order to achieve a well-informed decision. In such a context, the temporal reasoning facilities provided by TEMPORE are exploited in order to provide additional temporal information. In fact, (c) TEMPORE is used by the 'simulation' computerbased facility provided by GLARE (Terenziani et al. 2002a). When facing a decision, the userphysician might use such a 'simulation' facility in order to see the consequences of choosing the different alternative paths. The relevant pieces of information (e.g., resources, costs, time) can be gathered by the simulation facility from the alternative paths, and provided to the physician who can use them in order to compare the different alternatives. In particular, temporal reasoning can be useful in this context, in order to propagate the temporal constraints gathered from each path (e.g., in order to infer the maximal and minimal temporal duration of each path).

Last, but not least, temporal reasoning is used (during execution) (d) in order to help physicians to schedule the execution of clinical actions on the given patient. For example, the physician can exploit TEMPORE in order to see what are the temporal consequences of performing a given action at a given time. In other terms, given a pattern $\mathrm{A} 1, \ldots$, An of actions in a guideline, temporal reasoning can be used in order to answer queries such as 'If I perform action A1 today at 12 o'clock, when will I have to perform A2, .., An?', or 'Is it OK if I perform A1 today at 12, A2 at 18 and A3 at 20, and, if yes, when will I have to perform A4?'.

\subsection{Related works}

To the best of our knowledge, there is no other general purpose constraint-propagation-based approach which offers explicit support in order to deal with the temporal constraints raising from all the different issues we considered in this paper. In the following, we mention some of the constraint-based temporal reasoning approaches facing some of such issues which seem to us more closely related to our work.

In Morris et al.'s approach $(1993,1995,1996)$ only qualitative constraints between repeated events are dealt with. Repeated events are dealt with as 'classes' of events, and different quantifiers are used to relate them. As regards instances of events, Morris et al. (1995) introduced the notion of consistent scenario and used it in a 'predictive' perspective, sketching an algorithm for generating a consistent scenario of instances which are consistent with a knowledge base of temporal constraints between repeated events.

Loganantharaj mainly faced the problem of associating possibilistic distributions to qualitative temporal constraints between periodic events (Loganantharaj and Gimbrone 1995) and to metric constraints concerning the durations of events, which are also expressed using transition rules (Loganantharaj and Kurkovsky 1997, Loganantharaj and Gimbrone 1997). Such constraints are used in a 'predictive' way: temporal reasoning is used for projecting the constraints on the durations in the future using the current domain information.

In our previous work, we defined a high-level language to deal with user-defined periodicity (Terenziani 2003a) and with period-dependent qualitative temporal constraints between repeated events (which are coped with as 'classes' of events) and constraint propagation algorithms operating on them (Terenziani 1997, 2003b). We also proposed an initial algorithm to check the consistency of a knowledge base of such constraints with a set of instances of events exactly located in time (Terenziani 2000). Afterwards, we started to approach the problem of integrating 
both qualitative and quantitative constraints between classes and instances of events in a more general perspective, but not supporting periodic and composite events (Terenziani 2002, Terenziani and Anselma 2004), and proposed an initial approach to cope with qualitative and quantitative and periodic repetition constraints between classes of events in clinical guidelines (Terenziani et al. 2002a, b, Terenziani and Anselma 2003).

\subsection{Final remarks and future work}

We believe that our approach is significantly more expressive than related approaches in the literature. Although it finally relies on a low-level implementation based on standard STP, it builds on it a high-level language for temporal constraints which is significantly more expressive than STP (which only copes with distances between points), as needed whenever one wants to pair a Temporal Reasoner to more general systems coping with classes and instances of (possibly periodically repeated) events, as in the case of clinical guideline systems. Despite its expressiveness, TEMPORE is currently based on some limiting assumptions, some of which we aim to remove in our future work. However, we want to stress the point that most of these assumptions are needed in order to retain the tractability of the reasoning process, and are reasonable in real application domains (including the clinical guideline one: see section 6.1). We thus envision an extension of our approach along two mainstreams:

(1) 'conservative' extensions (i.e., extensions which still preserve the tractability of our approach);

(2) 'general' extensions, with the loss of tractability.

As concerns 'conservative' extensions, we are currently trying to extend our approach to cope also with repetitions based on conditions (e.g., repeat therapy $\mathrm{X}$ until condition $\mathrm{Y}$ holds). The treatment of conditioned repetitions is not critical at the level of classes of events (see (Terenziani et al. 2002b)), but its interplay with the checking of the consistency of instances wrt classes' constraints, considering the predictive role of classes' constraints, deserves specific attention. Furthermore, we are also planning to apply the 'local reasoning' properties we proved in (Brusoni et al. 1995, Console and Terenziani 1999) to support efficient query answering in TEMPORE.

As regards 'general' extensions, the tractability of our current approach strongly relies on the assumptions that instances are totally ordered ${ }^{\text {tti }}$ and of 'full observability' (i.e., if an instance is not explicitly present in the knowledge base, it didn't occur). Although both assumptions are generally reasonable in the clinical guideline context (which constitutes our main application area), we aim at developing a domain-independent approach covering also domains where such assumptions do not hold.

Roughly speaking, the lack of total ordering between instances and the lack of complete observability have on the reasoning mechanism basically the same effect: associating instances with the corresponding repetitions becomes a combinatorial problem ${ }^{*}$. In this perspective, we look at our current approach as the 'core' of a generalized approach in which extensive

itt Actually, total ordering is only required considering correlated instances of the same class. We also suppose that two such instances cannot occur exactly at the same time. Notice that our current approach covers also the case where ordering emerges from the initial propagation of the instances' constraints - without considering classes' constraints.

For example, as an extreme case, given a specific observed instance I of a repeated event E, if we have no temporal information about when I occurred, we have no means to understand if $I$ is the first, the second, etc. repetition of $E$, and we have to cope with all possible cases. Analogously, if E must be repeated x times, and we have just x-1 (or, even worst, $\mathrm{x}-\mathrm{i}$ ) instances of $\mathrm{E}$ (not ordered in time), and we do not have full observability, we have to cover all possible alternatives (i.e., the missing instance may correspond to the first repetition, to the second, etc.). 
backtracking (or backjumping) techniques are used to cope with the combinatorial explosion, and we are investigating how and when temporal constraints in the knowledge base can be used in order to bound the explosion (e.g., with an early pruning of temporally inconsistent alternatives) and/or to optimize the backtracking algorithm.

\section{Appendix A: Semantics of repetition/periodicity constraints}

For the sake of clarity, in the formula we denote by $\underline{F T}, \underline{A T+D T_{i}}, \underline{A T_{i}}, \underline{D T_{i}}, \underline{I T_{i}, j}$ the specific time intervals characterizing the repetition constraints (see Section 3.3.1), whose duration is $F T$, $A T+D T, A T, D T, I T$, respectively.

Let us denote with CNSduring, Cstarts and Cmeets the qualitative relations between classes of non-strict during, start and meet; let $n A T D T$ be the number of intervals $\underline{A T+D T}$ in the $\underline{F T}$, i.e., $n A T D T=\left\lfloor\frac{F T}{A T+D T}\right\rfloor$, and $n I T$ the number of $\underline{\mathrm{IT}} \mathrm{s}$ in an $\underline{\mathrm{AT}}$, i.e., $n I T=\left\lfloor\frac{A T}{I T}\right\rfloor$, then:

Repetition_Periodicity(FT, AT, DT, IT, freq, C) $\Leftrightarrow$

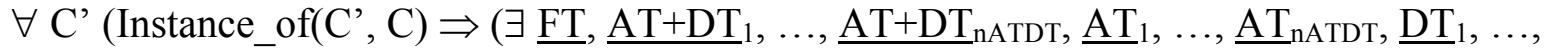
$\underline{\mathrm{DT}}_{\mathrm{nATDT}}, \underline{\mathrm{IT}}_{1,1}, \ldots, \underline{\mathrm{IT}}_{\mathrm{nATDT}, \mathrm{nIT}}, \underline{\text { freq }}_{1,1,1}, \ldots$, freq $_{\mathrm{nATDT}, \mathrm{nIT}, \text { freq }}, \mathrm{C}_{1,1,1}, \ldots, \mathrm{C}_{\mathrm{nATDT}, \mathrm{nIT}, \text { freq }}$ $\%$ durations $\%$

Cduration $(\underline{\mathrm{FT}}, \mathrm{FT}, \mathrm{FT}) \wedge$

$\forall \mathrm{i}=1, \ldots$, nATDT $\left(\right.$ Cduration $\left(\underline{\mathrm{AT}}+\mathrm{DT}_{\mathrm{i}}, \mathrm{AT}+\mathrm{DT}, \mathrm{AT}+\mathrm{DT}\right) \wedge \operatorname{Cduration}\left(\underline{\mathrm{AT}}_{\mathrm{i}}, \mathrm{AT}, \mathrm{AT}\right)$

$\left.\wedge \operatorname{Cduration}\left(\underline{D T}_{i}, \mathrm{DT}, \mathrm{DT}\right) \wedge \forall \mathrm{j}=1, \ldots, \operatorname{nIT}\left(\operatorname{Cduration}\left(\underline{\mathrm{IT}}_{\mathrm{i}, j}, \mathrm{IT}, \mathrm{IT}\right)\right)\right) \wedge$

$\%$ containment $\%$

$\forall \mathrm{i}=1, \ldots, \operatorname{nATDT}\left(\mathrm{CNSduring}\left(\underline{\mathrm{AT}+\mathrm{DT}_{\mathrm{i}}}, \mathrm{FT}\right) \wedge \mathrm{CNSduring}\left(\underline{\mathrm{AT}_{i}}, \underline{\left.\mathrm{AT}+\mathrm{DT}_{\mathrm{i}}\right)} \wedge \mathrm{CNSduring}\left(\underline{\mathrm{DT}_{\mathrm{i}}}\right.\right.\right.$, $\underline{\left.\mathrm{AT}+\mathrm{DT}_{\mathrm{i}}\right) \wedge}$

$\forall \mathrm{j}=1, \ldots$, nIT $\left(\mathrm{CNSduring}\left(\underline{\mathrm{IT}}_{\mathrm{i}}, \underline{\mathrm{AT}}_{\mathrm{i}}\right) \wedge\right.$

$\forall \mathrm{k}=1, \ldots$, freq $\left.\left.\left(\mathrm{CNSduring}\left(\underline{\text { freq }}_{\mathrm{i}, \mathrm{j}, \mathrm{k}}, \underline{\mathrm{IT}_{\mathrm{i}, \mathrm{j}}}\right) \wedge \mathrm{CNSduring}\left(\mathrm{C}_{\mathrm{i}, \mathrm{j}, \mathrm{k}}, \underline{\text { freq }}_{\mathrm{i}, \mathrm{j}, \mathrm{k}}\right)\right)\right)\right) \wedge$

$\%$ anchor to the start \%

$\operatorname{Cstarts}\left(\underline{\mathrm{AT}+\mathrm{DT}_{1}}, \underline{\mathrm{FT}}\right) \wedge$

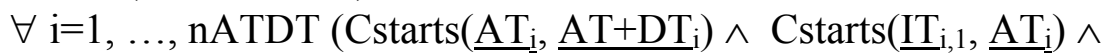

$\left.\forall \mathrm{j}=1, \ldots, \operatorname{nIT}\left(\operatorname{Cstarts}\left(\underline{\text { req }}_{\mathrm{i}, \mathrm{j}, 1}, \underline{\mathrm{IT}}_{\mathrm{i}, \mathrm{j}}\right)\right)\right) \wedge$

$\%$ ordering and covering $\%$

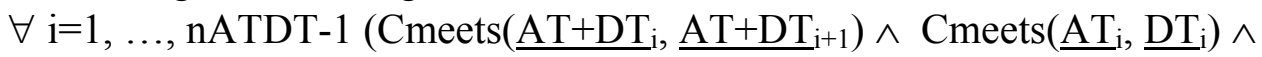

$\forall \mathrm{j}=1, \ldots, \operatorname{nIT}-1\left(\operatorname{Cmeets}\left(\underline{\mathrm{IT}}_{\mathrm{i}, \mathrm{j}}, \underline{\mathrm{IT}}_{\mathrm{i}, \mathrm{j}+1}\right) \wedge\right.$

$\forall \mathrm{k}=1, \ldots$, freq-1 $\left(\right.$ Cmeets $\left.\left.\left.\left(\underline{\text { freq }}_{\mathrm{i}, \mathrm{j}, \mathrm{k}}, \underline{\text { freq }}_{\mathrm{i}, \mathrm{j}, \mathrm{k}+1}\right)\right)\right)\right) \wedge$

$\%$ instances $\%$

$\forall \mathrm{i}=1, \ldots, \mathrm{nATDT}, \mathrm{j}=1, \ldots, \mathrm{nIT}, \mathrm{k}=1, \ldots$, freq (Instance_of $\left(\mathrm{C}^{\prime}, \mathrm{C}_{\mathrm{i}, \mathrm{j}, \mathrm{k}}\right)$ )

)$)$ )

\section{References}

S. Adali, L. Console, M.L. Sapino, M. Schenone and P. Terenziani, "Representing and reasoning with temporal constraints in multimedia presentations", in TIME 2000, Best Paper, Cape Breton, Canada, IEEE Press, pp. 3-11, 2000.

J.F. Allen, "Maintaining knowledge about temporal intervals", Communications of the ACM, 26(11): 832-843, 1983.

J.F. Allen, "Towards a general theory of action and time", Artificial Intelligence, 23: 123-154, 1984.

J. Allen, "Time and time again: the many ways to represent time", Int'l J. Intelligent Systems, 6(4):341-355, 1991. 
J.F. Allen and P. Hayes, "Moments and points in an interval-based temporal logic", Computational Intelligence 5: 225-238, 1989.

C. Bettini and R. De Sibi, "Symbolic Representation of User-defined Time Granularities", in TIME'99, IEEE Computer Society, pp. 17-28, 1999.

C. Bettini, S. Jajodia and X. Wang, "Solving multi-granularity constraint networks", Artificial Intelligence, 140(1-2):107-152, 2002.

V. Brusoni, L. Console and P. Terenziani, "On the computational complexity of querying bounds on differences constraints", Artificial Intelligence, 74(2):367-379, 1995.

V. Brusoni, L. Console, E. Lamma, P. Mello, M. Milano and P. Terenziani, "Resource-based vs. task-based approaches for scheduling problems", in 9th International Symposium on

Methodologies for Intelligent Systems, Zakopane, Poland, 1996.

V. Brusoni, L. Console, B. Pernici and P. Terenziani, "Later: managing temporal information efficiently", IEEE Expert, 12(4):56-64, 1997.

C. Combi, M. Franceschet and A. Peron, "Representing and Reasoning about Temporal

Granularities", Journal of Logic and Computation, 14(1):51-77, 2004.

L. Console and P. Terenziani, "Efficient processing of queries and assertions about qualitative and quantitative temporal constraints", Computational Intelligence, 15(4):442-465, 1999.

D. Cukierman and J. Delgrande, "Expressing time intervals and repetition within a formalization of calendars", Computational Intelligence, 14(4):563-597, 1998.

T. Dean and D. McDermott, "Temporal data base management", Artificial Intelligence, 32:1-56, 1987.

R. Dechter, I. Meiri and J. Pearl, “Temporal constraint networks”, Artificial Intelligence, 49:6195, 1991.

M. I. A. Galipienso and F. Barber Sanchís, "Representation and reasoning with disjunctive temporal constraints", in Ninth International Symposium on Temporal Representation and reasoning, IEEE Press, pp. 46-48, 2002.

P. Ladkin, "Primitive and units for time specification", in fifth National Conf. on Artificial Intelligence, Philadelphia, PA, pp. 354-359, 1986.

G. Ligozat, "On generalized Interval Calculi", in ninth National Conf. on Artificial Intelligence, pp. 234-240, 1991.

R. Loganantharaj and S. Gimbrone, "Probabilistic approach for representing and reasoning with repetitive events", in second International Workshop on Temporal Representation and Reasoning (TIME'95), Melbourne, FL, pp. 26-30, 1995.

R. Loganantharaj and S. Gimbrone, "Issues on synchronizing when propagating temporal constraints", in National Conference on Artificial Intelligence Workshop on Spatial and Temporal Reasoning, 1997.

R. Loganantharaj and S. Kurkovsky, “A new model for projecting temporal distance using fuzzy temporal constraints", in IEA/AIE'97, 1997.

I. Meiri, "Combining qualitative and quantitative constraints in temporal reasoning", in $A A A I^{\prime} 91$, pp. 260-267, 1991.

R.A. Morris, W.D. Shoaff and L. Khatib, "Path consistency in a network of non-convex intervals", in thirteenth Int'l Joint Conf. on Artificial Intelligence, Chambery, France, pp. 655-660, 1993.

R.A. Morris, L. Khatib and G. Ligozat, "Generating scenarios from specifications of repeating events", in second International Workshop on Temporal Representation and Reasoning (TIME'95), Melbourne, FL, pp. 41-48, 1995.

R.A. Morris, W.D. Shoaff and L. Khatib, "Domain independent temporal reasoning with recurring events", Computational Intelligence, 12(3):450-477, 1996.

I. Navarrete, A. Sattar and R. Marin, "Deciding consistency of a point-duration network with metric constraints”, in TIME'03, pp. 147-154, 2003. 
B. Nebel, "Reasoning and Revision in Hybrid Representation Systems", LNCS 422 (SpringerVerlag), 1990.

P. Terenziani, "Integrating calendar-dates and qualitative temporal constraints in the treatment of periodic events", IEEE Trans. on Knowledge and Data Engineering, 9(5), 1997.

P. Terenziani, "Integrated temporal reasoning with periodic events", Computational Intelligence, 16(2):210-256, 2000.

P. Terenziani, "Temporal reasoning with classes and instances of events", in TIME 2002, Manchester, UK, IEEE Press, pp. 100-107, 2002.

P. Terenziani, "Symbolic user-defined periodicity in temporal relational databases", IEEE

Transactions on Knowledge and Data Engineering, 15(2):489-509, 2003a.

P. Terenziani, "Towards a comprehensive treatment of temporal constraints about periodic events", International Journal of Intelligent Systems, 18(4):429-468, $2003 \mathrm{~b}$.

P. Terenziani and L. Anselma, "Towards a temporal reasoning approach dealing with instance-of, part-of and periodicity", in TIME-ICTL 2003, Cairns, Australia, IEEE Press, pp. 37-46, 2003.

P. Terenziani and L. Anselma, "A knowledge server for reasoning about temporal constraints between classes and instances of events". International Journal of Intelligent Systems, 19(10):919947, Wiley, 2004.

P. Terenziani, G. Molino and M. Torchio, "A modular approach for representing and executing clinical guidelines", Artificial Intelligence in Medicine, 23:249-276, 2001.

P. Terenziani, S. Montani, C. Carlini, G. Molino and M. Torchio, "Supporting physicians in taking decisions in clinical guidelines: the GLARE's 'what if' facility", Journal of the American Association of Medical Informatics (JAMIA), Proc. Annual Fall Symposium, 2002a.

P. Terenziani, C. Carlini and S. Montani, "Towards a comprehensive treatment of temporal constraints in clinical guidelines", in TIME 2002, Manchester, UK, IEEE Press, pp. 20-27, 2002 b. P. Terenziani, S. Montani, M. Torchio, G. Molino and L. Anselma, "Temporal consistency checking in clinical guidelines acquisition and execution: the GLARE's approach", Journal of the American Medical Informatics Association (JAMIA), Special issue on the Fall AMIA 2003 Symposium, pp. 659-663, 2003.

P. Van Beek, "Temporal query processing with indefinite information", Artificial Intelligence in Medicine, 3:325-339, 1991.

F. Van Eynde, "Iteration, habituality and verb form semantics", in third Conference of European Chapter of the Association for Computational Linguistics, Copenhagen, Denmark, pp. 270-277, 1987.

L. Vila, "A survey on temporal reasoning in Artificial Intelligence", AI Communications, 7(1):428, 1994.

M. Vilain, H. Kautz and P. VanBeek, "Constraint propagation algorithms for temporal reasoning: a revised report", In D.S. Weld and J. deKleer (eds), Readings in Qualitative Reasoning about Physical Systems (Morgan Kaufmann), pp. 373-381, 1990. 


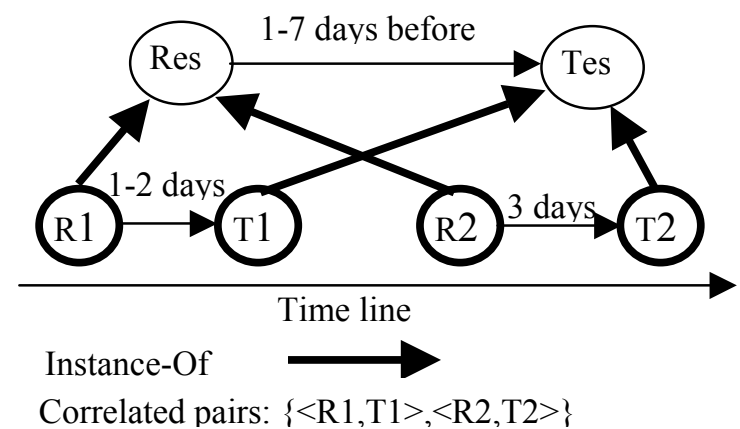

Figure 1. Temporal constraints between classes (Res and Tes) and instances $(R 1, R 2, T 1$, and $T 2)$ of events.

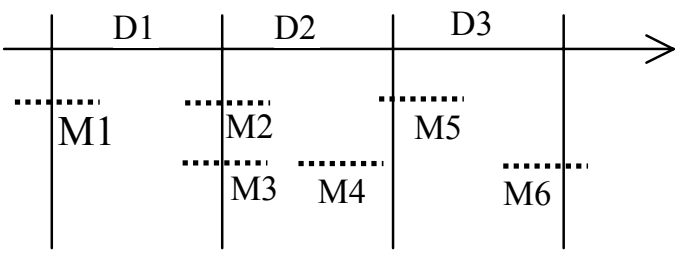

Figure 2. Association of actions with days.

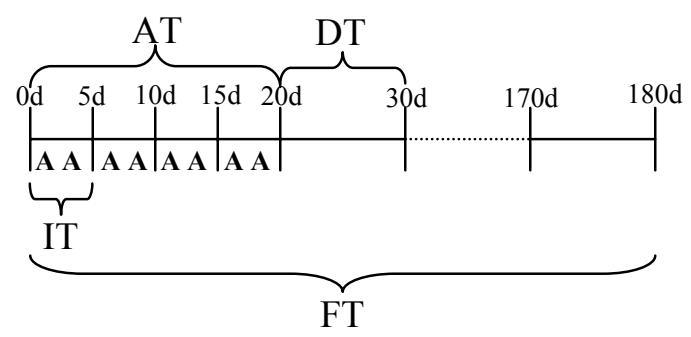

Figure 3. Representation of periodicity for Ex.5. A's represent instances of the action A. 


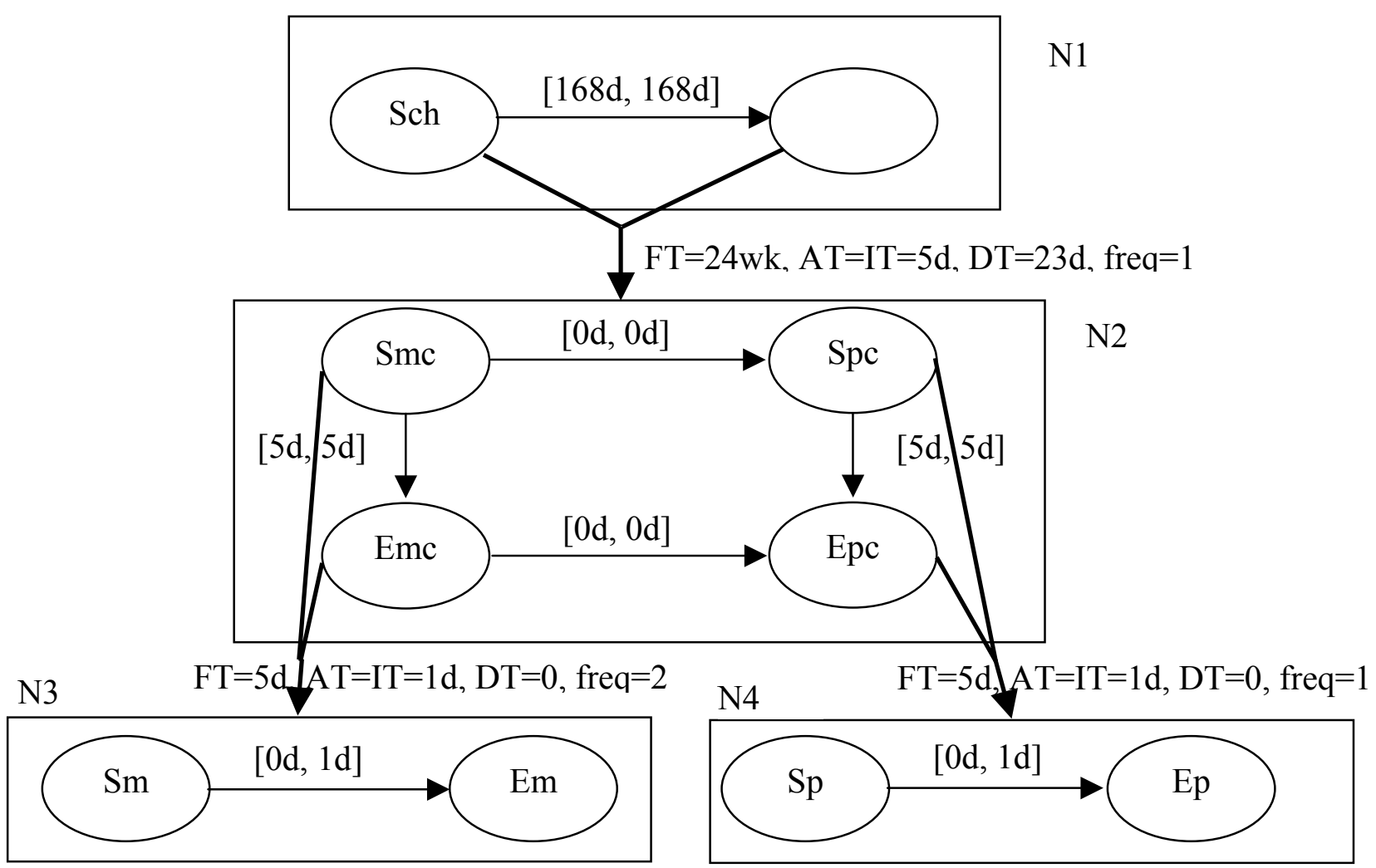

Figure 4. A naïve graphical representation of the STP-tree for the multiple mieloma chemotherapy guideline. Thin lines and edges between nodes in an STP represent bound on differences constraints. Thick edges from a pair of nodes to a child STP represent repetitions. The overall therapy (node N1) is composed of 6 cycles of 5 days plus a delay of 23 days (for a global frame time of 24 weeks). In each cycle (node N2), two therapies are executed in parallel: melphalan (node N3: Sm and Em are the starting and ending nodes), to be repeated twice a day, and prednisone (node $N 4: S p$ and $E p$ are the starting and ending nodes), to be repeated once a day. Edges between any two nodes $X$ and $Y$ in an STP (say $N 2$ ) of the STP-tree are labeled by a pair $[n, m]$ representing the minimal and maximal distance between $X$ and $Y$. 
function STP_tree_consistency(X: STP, FT: duration, AT: duration, DT: duration, IT: duration, freq: integer) : STP

(1) if $\operatorname{not}((\mathrm{AT}+\mathrm{DT}) \subseteq \mathrm{FT})$ or $\operatorname{not}(\mathrm{IT} \subseteq \mathrm{AT})$ then return INCONSISTENT

(2) $\mathrm{Y} \leftarrow$ FloydWarshall $(\mathrm{X})$;

(3) if $Y=I N C O N S I S T E N T$ then return INCONSISTENT

(4) let min the maximum between the minimum distances between all the points in $Y$

(5) $\mathrm{Max} \leftarrow$ IT $-($ freq -1$) * \min$

(6) for each pair of points $p_{i}$ and $p_{j}(i \neq j)$ in $Y$ do

(7) impose (in $\mathrm{Y}$ ) that the maximum distance between $\mathrm{p}_{\mathrm{i}}$ and $\mathrm{p}_{\mathrm{j}}$ is the minimum between the current maximum distance in $\mathrm{X}$ and Max od

(8) $\mathrm{Y} \leftarrow$ FloydWarshall( $\mathrm{Y})$

(9) if $\mathrm{Y}=\mathrm{INCONSISTENT}$ then return INCONSISTENT else return $\mathrm{Y}$

Figure 5. Algorithm for checking consistency of an STP-node. 
function unfoldClasses(T : CKB) : STP

(1) initialize S to an empty STP

(2) add to $\mathrm{S}$ the placeholder class $\mathrm{C}_{\mathrm{T}}$

(3) unfoldSTPNode(root(T), $\left.\mathrm{C}_{\mathrm{T}}, \mathrm{S}\right)$

(4) $\mathrm{S}^{\prime} \leftarrow$ FloydWarshall( $(\mathrm{S})$

(5) return $S$ '

procedure unfoldSTPNode(/*input*/ X : STPNode, $\mathrm{C}_{\mathrm{X}}$ : Class, $/ *_{\text {input\&output*/ S : STP) }}$

(1) forall $C_{a} \mid C_{a}$ is not a repeated class in $X$ do

(2) copy the class $C_{a}$ in $S$

(3) add to $\mathrm{S}$ the constraints that $\mathrm{C}_{\mathrm{a}} \subseteq \mathrm{C}_{\mathrm{X}}$ od

(4) forall $C_{r} \mid C_{r}$ is a repeated class in $X$ do

(5) add to $\mathrm{S}$ the placeholder class $\mathrm{FT}_{\mathrm{Cr}}$

(6) $/ *$ let FT, AT, DT, IT and freq the periodicity parameters for class $\mathrm{C}_{\mathrm{r}} * /$

(7) unfoldRep $\left(\mathrm{X}, \mathrm{C}_{\mathrm{r}}, \mathrm{FT}, \mathrm{AT}, \mathrm{DT}\right.$, IT, freq, $\left.\mathrm{FT}_{\mathrm{Cr}}, \mathrm{S}\right)$

(8) add to $\mathrm{S}$ the constraints that $\mathrm{FT}_{\mathrm{Cr}} \subseteq \mathrm{C}_{\mathrm{X}}$ od

(9)for each monadic constraint in X do add to $\mathrm{S}$ the constraint wrt the corresponding classes in $\mathrm{S}$

(10)for each binary constraint in $\mathrm{X}$ do add to $\mathrm{S}$ the constraint wrt the corresponding classes in $\mathrm{S}$

procedure unfoldRep $\left(/ *_{\text {input* }}\right.$ X : STPNode, C : Class, FT: duration, AT: duration, DT: duration, IT: duration, freq: integer, $\mathrm{FT}_{\mathrm{C}}$ : Class, $/ *$ input\&output*/ S : STP)

(1) for all $\mathrm{ATDT}_{\mathrm{i}}$ 's that fit in $\mathrm{FT}_{\mathrm{C}} / *$ i.e. for $[\mathrm{FT} /(\mathrm{AT}+\mathrm{DT})\rfloor$ times $* /$ do

(2) add to $\mathrm{S}$ the placeholder class $\mathrm{ATDT}_{\mathrm{i}}$

(3) add to $\mathrm{S}$ the placeholder classes $\mathrm{AT}_{\mathrm{i}}$ and $\mathrm{DT}_{\mathrm{i}}$

(4) for all $\mathrm{IT}_{\mathrm{ij}}$ 's that fit in $\mathrm{AT}_{\mathrm{i}} / *$ i.e. for $\lfloor\mathrm{AT} / \mathrm{IT}\rfloor$ times $* /$ do

(5) add to $\mathrm{S}$ the placeholder class $\mathrm{IT}_{\mathrm{ij}}$

(6) for all freq $\mathrm{ijk}_{\mathrm{jk}}$ 's that fit in $\mathrm{IT}_{\mathrm{ij}} / *$ i.e. for freq times $* /$ do

(7) add to $\mathrm{S}$ the placeholder class freq $\mathrm{q}_{\mathrm{ijk}}$

(8) unfoldSTPNode(Child(C,X), freq $\left.q_{i j k}, S\right)$ od od od

(9) add to $\mathrm{S}$ the constraints that the durations of $\mathrm{FT}_{\mathrm{C}}, \mathrm{ATDT}_{\mathrm{i}}, \mathrm{AT}_{\mathrm{i}}, \mathrm{DT}_{\mathrm{i}}$ and $\mathrm{IT}_{\mathrm{j}}$ are FT, AT+DT, AT, DT and IT, respectively

(10)add to $\mathrm{S}$ the constraints that $\mathrm{ATDT}_{\mathrm{i}}, \mathrm{AT}_{\mathrm{i}}$ and $\mathrm{DT}_{\mathrm{i}}, \mathrm{IT}_{\mathrm{ij}}$ and freq $\mathrm{ijk}_{\mathrm{ijk}}$ are contained in $\mathrm{FT}_{\mathrm{C}}, \mathrm{ATDT}_{\mathrm{i}}, \mathrm{AT}_{\mathrm{i}}$ and $\mathrm{IT}_{\mathrm{ij}}$, respectively

(11) add to $\mathrm{S}$ the constraints that $\mathrm{ATDT}_{1}$ starts $\mathrm{FT}_{\mathrm{C}}, \mathrm{AT}_{\mathrm{i}}$ starts $\mathrm{ATDT}_{\mathrm{i}}, \mathrm{IT}_{\mathrm{i} 1}$ starts $\mathrm{AT}_{\mathrm{i}}$, and freq $q_{\mathrm{ij} 1}$ starts $\mathrm{IT}_{\mathrm{ij}}$

(12) add to $\mathrm{S}$ the constraints that $\mathrm{ATDT}_{\mathrm{i}}$ meets $\mathrm{ATDT}_{\mathrm{i}+1}, \mathrm{AT}_{\mathrm{i}}$ meets $\mathrm{DT}_{\mathrm{i}}, \mathrm{IT}_{\mathrm{ij}}$ meets $_{\mathrm{IT}} \mathrm{T}_{\mathrm{ij}+1}$, and freq $\mathrm{q}_{\mathrm{ijk}}$ meets freq $_{\mathrm{ijk}+1}$

procedure integratedConsistency $(\mathrm{T}: \mathrm{CKB}, \mathrm{I}: \mathrm{IKB})$

(1) $\mathrm{S} \leftarrow$ unfoldClasses $(\mathrm{T})$

(2) add to I the placeholder instances corresponding to the placeholder classes in $\mathrm{S}$

(3) add to I the placeholder instances corresponding to the non-atomic, non-repeated classes in S

(4) I' $\leftarrow$ FloydWarshall(I)

(5) if I'=INCONSISTENT then return INCONSISTENT

(6) for each class $\mathrm{C}$ in $\mathrm{S}$ in temporal order do

(7) let $\mathrm{i}$ be the first instance of $\mathrm{C}$ in I' not yet taken in consideration

(8) if $i$ does not exist then return INCONSISTENT od

(9) if there exists an instance in I' not yet considered then return INCONSISTENT

(10) forall instance $\mathrm{E} 1$ and class $\mathrm{C} 1$ such that Instance of $(\mathrm{E} 1, \mathrm{C} 1)$ holds and $\mathrm{t} \leq \mathrm{End}(\mathrm{C} 1)-\operatorname{Start}(\mathrm{C} 1) \leq \mathrm{u}$ is a constraint in $\mathrm{S}$ do add the constraint $\{\mathrm{t} \leq \mathrm{End}(\mathrm{E} 1)-\mathrm{Start}(\mathrm{E} 1) \leq \mathrm{u}\}$ in I' od

(11) forall correlated instances $\mathrm{E} 1, \mathrm{E} 2, \mathrm{E} 1 \neq \mathrm{E} 2$ do

- let $\mathrm{C} 1, \mathrm{C} 2$ the classes corresponding to $\mathrm{E} 1$ and $\mathrm{E} 2$

- instantiate on E1 and E2 the constraints in S between $\mathrm{C} 1$ and $\mathrm{C} 2$

od

(12)I" $\leftarrow$ FloydWarshall(I')

(13)if I"=INCONSISTENT then return INCONSISTENT 
Figure 6. Algorithm for checking consistency of classes+instances. 
procedure integratedConsistencyNoFuture(T : CKB, I : IKB, $\underline{\mathbf{R T}})$

(1) $\mathrm{S} \leftarrow$ unfoldClasses(T)

(1a) for each instance $i$ in I do

(1b) add the constraint the i starts before RT

(1c)hypothesizedInstances $\leftarrow \varnothing$

(2) add to I the placeholder instances corresponding to the placeholder classes in $\mathrm{S}$

(3) I' $\leftarrow$ FloydWarshall(I)

(4) if I' $=$ INCONSISTENT then return INCONSISTENT

(5) for each class $\mathrm{C}$ in $\mathrm{S}$ with start times in ascending order do

(6) let $\mathrm{i}$ be the first instance of $\mathrm{C}$ in I' not yet taken in consideration

(7) if $\mathrm{i}$ does not exist then

(7a) add to I a new instance i' of C

(7b) hypothesizedInstances $\leftarrow$ hypothesizedInstances $\cup\left\{i^{\prime}\right\}$ od

(8) if there exists an instance in I' not yet considered then return INCONSISTENT

(9) inherit the monadic constraints from the classes in $\mathrm{S}$ to the corresponding instances in I'

(10)inherit the binary constraints from the classes in S to the corresponding instances in I'

(11)I" $\leftarrow$ FloydWarshall(I')

(12)if I"=INCONSISTENT then return INCONSISTENT

(13)for each $\mathrm{i} \in$ hypothesizedInstances do if NEC(Start(i) before RT) then return INCONSISTENT

Figure 7. Algorithm for checking consistency of classes+instances with no observability in the future.

\begin{tabular}{|c|c|}
\hline \# of (unfolded) classes & Time (ms) \\
\hline 10 & 10 \\
\hline 50 & 63 \\
\hline 100 & 656 \\
\hline 200 & 3937 \\
\hline 500 & 72885 \\
\hline 1000 & 492821 \\
\hline
\end{tabular}

Table 1. Number of unfolded classes $L$ and CPU times. 